\title{
Diffusion tensor imaging in metachromatic leukodystrophy
}

\author{
Diane F. van Rappard ${ }^{1,2} \cdot$ Marsh Königs ${ }^{3,4} \cdot$ Marjan E. Steenweg $^{1,2}$. Jaap Jan Boelens ${ }^{5}$. Jaap Oosterlaan ${ }^{3,6}$. \\ Marjo S. van der Knaap ${ }^{1,2,7} \cdot$ Nicole I. Wolf ${ }^{1,2}$. Petra J. W. Pouwels ${ }^{2,8}$
}

Received: 23 November 2017 / Revised: 9 January 2018 / Accepted: 23 January 2018 / Published online: 30 January 2018

(c) The Author(s) 2018. This article is an open access publication

\begin{abstract}
Objective We aimed to gain more insight into the pathomechanisms of metachromatic leukodystrophy (MLD), by comparing magnitude and direction of diffusion between patients and controls at diagnosis and during follow-up.

Methods Four late-infantile, 16 juvenile and 8 adult onset MLD patients [of which 13 considered eligible for hematopoietic cell transplantation (HCT)] and 47 controls were examined using diffusion tensor imaging. Fractional anisotropy (FA), mean diffusivity (MD), axial diffusivity (AD) and radial diffusivity (RD) were quantified and compared between groups using tract-based spatial statistics (TBSS). Diffusion measures were determined for normal-appearing white matter (NAWM), corpus callosum, thalamus (all based on subject-wise segmentation), and pyramidal tracts, determined with probabilistic tractography. Measures were compared between HCT-eligible patients, non-eligible patients and controls using general linear model and nonparametric permutation analyses (randomise) for TBSS data, considering family-wise error corrected $p<0.05$ significant.

Results Throughout white matter (WM), FA was decreased and MD and RD increased in both patient groups compared to controls, while AD was decreased in NAWM and corpus callosum. In the thalamus, no differences in FA were observed, but all diffusivities were increased in both patient groups. Differences were most pronounced between controls and patients non-eligible for HCT. Longitudinally (median follow-up 3.9 years), diffusion measures remained relatively stable for HCTtreated patients, but were progressively abnormal for non-eligible patients.

Interpretation The observed diffusion measures confirm that brain microstructure is changed in MLD, reflecting different pathological processes including loss of myelin and sulfatide accumulation. The observation of both increased and decreased $\mathrm{AD}$ probably reflects a balance between myelin and axonal loss vs. intracellular sulfatide storage in macrophages, depending on region and disease stage.
\end{abstract}

Keywords Diffusion weighted imaging $\cdot$ Axial diffusivity $\cdot$ Radial diffusivity $\cdot$ Sulfatide storage $\cdot$ White matter disorder

\section{Introduction}

Metachromatic leukodystrophy (MLD, OMIM 250100) is an autosomal recessive lysosomal disorder caused by mutations in the ARSA gene. This results in deficiency of the enzyme arylsulfatase A (ASA), essential for sulfatide

Nicole I. Wolf and Petra J. W. Pouwels contributed equally to this work.

Electronic supplementary material The online version of this article (https://doi.org/10.1007/s00415-018-8765-3) contains supplementary material, which is available to authorized users.

Petra J. W. Pouwels

pjw.pouwels@vumc.nl

Extended author information available on the last page of the article metabolism [1]. Sulfatides are major myelin lipids; their accumulation, mainly in membranes, leads to demyelination and subsequently storage in macrophages that cannot digest them [1]. MLD is a devastating disease: without treatment, eventually all acquired skills are lost and patients die.

MLD has three clinical subtypes, based on age of onset. The late-infantile form starts before 30 months, usually presenting with motor deterioration. The juvenile form presents with a combination of motor and cognitive decline before 16 years. The adult form begins with cognitive decline and psychiatric symptoms thereafter [2]. When performed early, hematopoietic cell therapy (HCT) has promising results, especially for juvenile and adult patients [3, 4]. 
Brain magnetic resonance imaging (MRI) in MLD is characterized by bilateral symmetric $\mathrm{T} 2$ signal hyperintensities, starting in the corpus callosum and subsequently involving periventricular, central and subcortical white matter (WM), in addition to projection fibers such as the corticospinal tract, and eventually cerebellar WM [5]. Thalamic volume and signal intensity on T2-weighted images in the thalamus are decreased already at diagnosis $[6,7]$. Typical for MLD are stripes of low signal intensity throughout the hyperintense signal on T2-weighted images in the cerebral WM, related both to the accumulation of macrophages bursting with undigested lipids and to better preserved perivascular myelin [8].

Brain diffusion tensor imaging (DTI) is based on the motion of water molecules, which is more restricted perpendicular to than along WM fibers, a feature termed diffusion anisotropy [9]. Magnitude and direction of diffusivity are determined by molecules, membranes and microtubules, and provide information about tissue composition and microstructure and its architectural organization [10, $11]$.

The tensor model is a relatively simple model using diffusion-weighted images (DWI) obtained with one $b$ value. It results in axial diffusivity (AD), radial diffusivity (RD) and the derived fractional anisotropy (FA) and mean diffusivity (MD) [11]. Often, increased RD is thought to be correlated with myelin degradation and changes in $\mathrm{AD}$ to axonal degeneration or inflammation and gliosis [12-17], but it is difficult to unequivocally associate the interpretation of diffusivity variations with specific biophysical changes [18].

The precise pathomechanisms involved in MLD, such as importance of inflammation or how accumulated sulfatides lead to demyelination, are not completely understood. DTI is, taking into account its recognized limitations, a valuable tool to gain more insight into changes in tissue properties in MLD. We, therefore, compared diffusion measures (FA and the three diffusivities) between patients who were eligible for HCT, patients not eligible at time of diagnosis, and controls. HCT-eligible patients are typically in an early disease stage, while patients not eligible for HCT have more advanced disease with extensive demyelination of the WM.
We also studied the longitudinal behavior of diffusion measures of both treated and untreated patients.

\section{Methods}

\section{Patients and control subjects}

All 28 MLD patients (4 late-infantile, 16 juvenile, and 8 adult onset), visiting the Center for Childhood White Matter Disorders, who underwent a quantitative MRI protocol at time of diagnosis between January 2007 and April 2017 were included in this retrospective study, in addition to 47 control subjects in the same age range (Table 1), after informed consent. The study was approved by the institutional review board. Diagnosis of MLD was established by brain MRI, ASA activity and ARSA mutation analysis [4]. Motor function was scored by the MLD Gross Motor Function (MLD-GMF) at baseline and at latest clinical follow-up [19]. Cognitive function was evaluated through neuropsychological examination using the Wechsler Intelligence scale for Children or Adults, as appropriate for age. Eligibility for HCT was based on patients' neurological examination (no major abnormalities and able to walk independently) and cognitive function (IQ > 75). Treatment with HCT was performed as described before [4]. Characteristics of individual patients are described in Table 2. Thirteen patients were considered eligible for HCT, and 15 non-eligible for HCT. Fourteen patients received HCT (2 patients initially classified as non-eligible; one eligible patient declined HCT) [4]. Follow-up MRI examinations were available for 17 patients (12 HCTeligible, 5 non-eligible). Median follow-up time between first and last MRI examination was 3.9 years (range 2.5 months to 11.2 years).

Control subjects at 1.5-T underwent MRI for reasons like mild developmental delay, headache; they had normal MRI and neurological examination. Controls at $3 \mathrm{~T}$ had experienced a non-neurological trauma and were included in a previous study [20].

Table 1 Demographic information

\begin{tabular}{llllll}
\hline & Controls & All MLD patients & Eligible for HCT & Not eligible for HCT & Contrasts $p$ \\
\hline Number of subjects & 47 & 28 & 13 & 15 & $\mathrm{~ns}$ \\
Male/female & $23 / 24$ & $9 / 19$ & $6 / 7$ & $3 / 12$ & $12.4(8.3)$ \\
Age at first scan (mean, SD, years) & $10.5(5.3)$ & $14.5(9.5)$ & $16.9(10.6)$ & $2 / 11 / 2$ & $0.017^{\mathrm{a}}$ \\
Late-infantile/juvenile/adult MLD & na & $4 / 16 / 8$ & $2 / 5 / 6$ & $\mathrm{~ns}$ \\
\hline
\end{tabular}

$n s$ not significant, $n a$ not applicable

${ }^{\text {a }}$ Post hoc Dunnett's T3 revealed no significant pairwise group differences 


\section{Acquisition}

Between January 2007 and April 2013, 19 patients and 20 controls had a baseline examination at $1.5 \mathrm{~T}$ (Siemens Sonata, Erlangen, Germany). Between May 2013 and April 2017, 9 patients and 27 controls had a baseline examination at $3 \mathrm{~T}$ (GE MR750, Milwaukee, WI, USA).

Conventional imaging included sagittal 3-dimensional (3D)-T1 and axial FLAIR, using the same spatial resolution at both field strengths [20,21]. FLAIR imaging was not performed for control subjects at $3 \mathrm{~T}$. DTI was obtained with a multi-slice echo planar imaging sequence and isotropic $2.5 \times 2.5 \times 2.5 \mathrm{~mm}^{3}$ voxels. At $1.5 \mathrm{~T}$, we obtained $1 \mathrm{~b} 0$ volume and 12 gradient directions with $b$ value $750 \mathrm{~s} / \mathrm{mm}^{2}$, 2 acquisitions, TR/TE 6700/81 ms [21]. At 3 T, we obtained $5 \mathrm{~b} 0$ volumes and 30 gradient directions with $b$ value $750 \mathrm{~s} /$ $\mathrm{mm}^{2}, 1$ acquisition, TR/TE 5100/75 ms, and parallel imaging factor 2 [20].

\section{Analysis}

Diffusion tensor imaging data were analyzed using FMRIB's software library FSL after correction of eddy current distortion and subject motion. The diffusion tensor was fitted resulting in maps of FA, AD, RD and MD. Tract-based spatial statistics (TBSS) was used to align FA images from all subjects into a common space and to create a mean FA skeleton. The aligned FA images of all participants were projected onto this skeleton and fed into voxel-wise crossparticipant statistics using randomise (see "Statistical analysis") [22].
Table 2 Characteristics of MLD patients

\begin{tabular}{|c|c|c|c|c|c|c|}
\hline Patient & MLD type & Age (years) & $\begin{array}{l}\text { Baseline } \\
\text { scan }(\mathrm{T})\end{array}$ & $\begin{array}{l}\text { Follow-up } \\
\text { scans }(n)\end{array}$ & $\begin{array}{l}\text { Eligible for } \\
\text { HCT }\end{array}$ & HCT-treated \\
\hline 045 & Late-infantile & 2.0 & 1.5 & $3^{\mathrm{b}}$ & Yes & Yes \\
\hline 050 & Late-infantile & 2.1 & 1.5 & $1^{\mathrm{b}}$ & Yes & Yes \\
\hline 057 & Late-infantile & 2.4 & 3 & 0 & No & No \\
\hline 026 & Late-infantile & 2.6 & 1.5 & 0 & No & No \\
\hline 016 & Juvenile & 6.5 & 1.5 & $3^{\mathrm{d}}$ & Yes & Yes \\
\hline 039 & Juvenile & 7.0 & 1.5 & $1^{\mathrm{c}}$ & No & No \\
\hline 029 & Juvenile & 7.1 & 1.5 & 0 & No & No \\
\hline 053 & Juvenile & 7.1 & 1.5 & $1^{\mathrm{b}}$ & No & Yes \\
\hline 005 & Juvenile & 7.2 & 1.5 & $1^{\mathrm{c}}$ & No & No \\
\hline 065 & Juvenile & 7.4 & 3 & $2^{\mathrm{c}}$ & Yes & Yes \\
\hline 064 & Juvenile & 8.6 & 3 & 0 & No & No \\
\hline 006 & Juvenile & 8.6 & 1.5 & $1^{\mathrm{c}}$ & No & No \\
\hline 054 & Juvenile & 12.5 & 1.5 & $1^{\mathrm{c}}$ & No & No \\
\hline 060 & Juvenile & 13.1 & 3 & 0 & No & No \\
\hline 058 & Juvenile & 13.8 & 3 & $3^{c}$ & Yes & Yes \\
\hline 014 & Juvenile & 14.1 & 1.5 & $4^{\mathrm{d}}$ & Yes & Yes \\
\hline 022 & Juvenile & 15.1 & 1.5 & 0 & No & No \\
\hline 067 & Juvenile & 17.6 & 3 & 0 & Yes & Yes \\
\hline 068 & Juvenile & 19.2 & 3 & 0 & No & No \\
\hline 061 & Juvenile & 20.1 & 3 & 0 & No & No \\
\hline 021 & Adult & 17.8 & 1.5 & $3^{\mathrm{d}}$ & Yes & Yes \\
\hline 051 & Adult & 22.5 & 1.5 & 0 & No & Yes \\
\hline 063 & Adult & 23.1 & 3 & $1^{\mathrm{c}}$ & Yes & Yes \\
\hline 041 & Adult & 25.4 & 1.5 & $6^{\mathrm{d}}$ & Yes & Yes \\
\hline 032 & Adult & 26.9 & 1.5 & $2^{\mathrm{d}}$ & Yes & No \\
\hline 002 & Adult & 28.4 & 1.5 & $5^{\mathrm{d}}$ & Yes & Yes \\
\hline 056 & Adult & 32.5 & 1.5 & 0 & No & No \\
\hline 015 & Adult & 35.2 & 1.5 & $4^{\mathrm{d}}$ & Yes & Yes \\
\hline
\end{tabular}

${ }^{\mathrm{a}}$ Age at baseline examination

${ }^{\mathrm{b}}$ Follow-up examinations at $1.5 \mathrm{~T}$

${ }^{c}$ Follow-up examinations at $3 \mathrm{~T}$

${ }^{\mathrm{d}}$ Follow-up examinations at both 1.5 and $3 \mathrm{~T}$ 
Based on the regional differences found in the TBSS analyses, we further analyzed diffusion measures in the following regions of interest (ROIs): normal-appearing white matter (NAWM, corpus callosum and thalamus in all subjects, and abnormal cerebral WM in patients. In addition, we analyzed the pyramidal tracts, which were determined for each subject by tractography between motor cortex and cerebral peduncles (see below).

To determine these ROIs in DTI subject space, we first outlined abnormal WM on 2D FLAIR images of patients using clusterize, a semiautomatic segmentation algorithm involving iterative region growing followed by interactive selection of abnormal WM clusters [23]. The mask of abnormal WM was registered to the corresponding 3DT1, and filled with signal intensity resembling NAWM [24]. This 3DT1 image was then segmented with the FSL tools FAST [25] and FIRST [26] to obtain WM, gray matter (GM) and deep GM (DGM) structures, including the thalamus. DGM and abnormal WM were subtracted from the WM mask to obtain NAWM. ROIs for corpus callosum and cerebral peduncles were identified using the Johns Hopkins University (JHU) WM atlas defined in standard Montreal Neurological Institute (MNI) space [27]. The motor cortex was identified in MNI space using the Automated Anatomical Labeling (AAL) atlas [28]. ROIs in MNI space were warped into 3DT1 subject space after linear and non-linear registration using FSL tools FLIRT and FNIRT. All ROIs were subsequently registered to DTI subject space using nearest neighbor interpolation.

The ROIs of motor cortex and cerebral peduncles were used as seed and target for probabilistic tractography using the FSL tools bedpostx and probtrackx 2 to obtain the left and right pyramidal tract [29]. Mean diffusion measures within the pyramidal tracts were determined by weighting the underlying FA and diffusivity maps by the probability of a voxel within the tract.

\section{Statistical analysis}

Statistical analyses were performed for HCT-eligible and non-eligible patients at baseline and control subjects. Groups were compared on demographic variables using ANOVA and Chi-square tests, as appropriate. In the TBSS analysis, FA, MD, AD and RD were compared among the three groups with nonparametric permutation analysis (randomise), using age and scanner as covariate. We considered a family-wise error (FWE) corrected $p<0.05$ significant.

General linear model analyses (ANOVA) including age and scanner as covariates were performed for a three-group comparison of diffusion measures at baseline within selected ROIs. In case of main group effects, we performed post hoc pairwise comparisons between groups, using Dunnett's T3.
To evaluate longitudinal evolution of disease, we created scatter plots of ROI-based diffusion measures as function of age, and we qualitatively described changes for patients with follow-up examinations.

The pyramidal tracts primarily regulate motor function. We, therefore, determined Spearman rank correlations between diffusion measures of all 28 patients at baseline and motor function, determined with MLD-GMF, at latest clinical follow-up. $p<0.05$ was considered significant.

\section{Results}

\section{Baseline}

Controls, HCT-eligible and non-eligible patients did not differ on demographic variables. Only for age, a main group effect was detected $(p=0.017)$, which was not reflected in post hoc testing (Table 1).

In the TBSS analysis of all baseline examinations (see Fig. 1), FA was decreased in HCT-eligible and non-eligible patients compared to controls, and in non-eligible patients compared to eligible patients in almost the entire skeleton. The differences were highly significant, since they remained present at FWE-corrected $p<0.001$, as indicated by the yellow color. An increase of MD and $\mathrm{RD}$ in both patient groups compared to controls was also observed in almost the whole skeleton, again highly significant (FWE-corrected $p<0.001$ ), as indicated by the light blue color. An increase of MD and RD in non-eligible patients compared to eligible patients was limited to a smaller part of the skeleton. An increase in AD in HCT-eligible patients compared to controls was restricted to part of the periventricular WM and the genu and splenium of the corpus callosum. In HCT-non-eligible patients compared to both controls and eligible patients, AD was increased mainly in the thalamus, but decreased in a large part of the skeleton, including the corpus callosum.

In the selected ROIs (see Fig. 2), FA was decreased for both patient groups compared to controls in NAWM, corpus callosum and pyramidal tracts. Differences were most pronounced between controls and HCT-non-eligible patients. The relative and absolute decrease in FA was largest in corpus callosum. In patients, FA in abnormal WM was smaller than in NAWM, and lower in HCT-noneligible patients than in eligible patients (supplementary material, Table 1). Although the TBSS analysis showed group differences in FA in the skeletonized thalamus, there were no FA differences in the thalamus based on a ROI analysis. The FA observations in all ROIs were replicated when splitting the groups based on field strength (supplementary material, Fig. 1). 


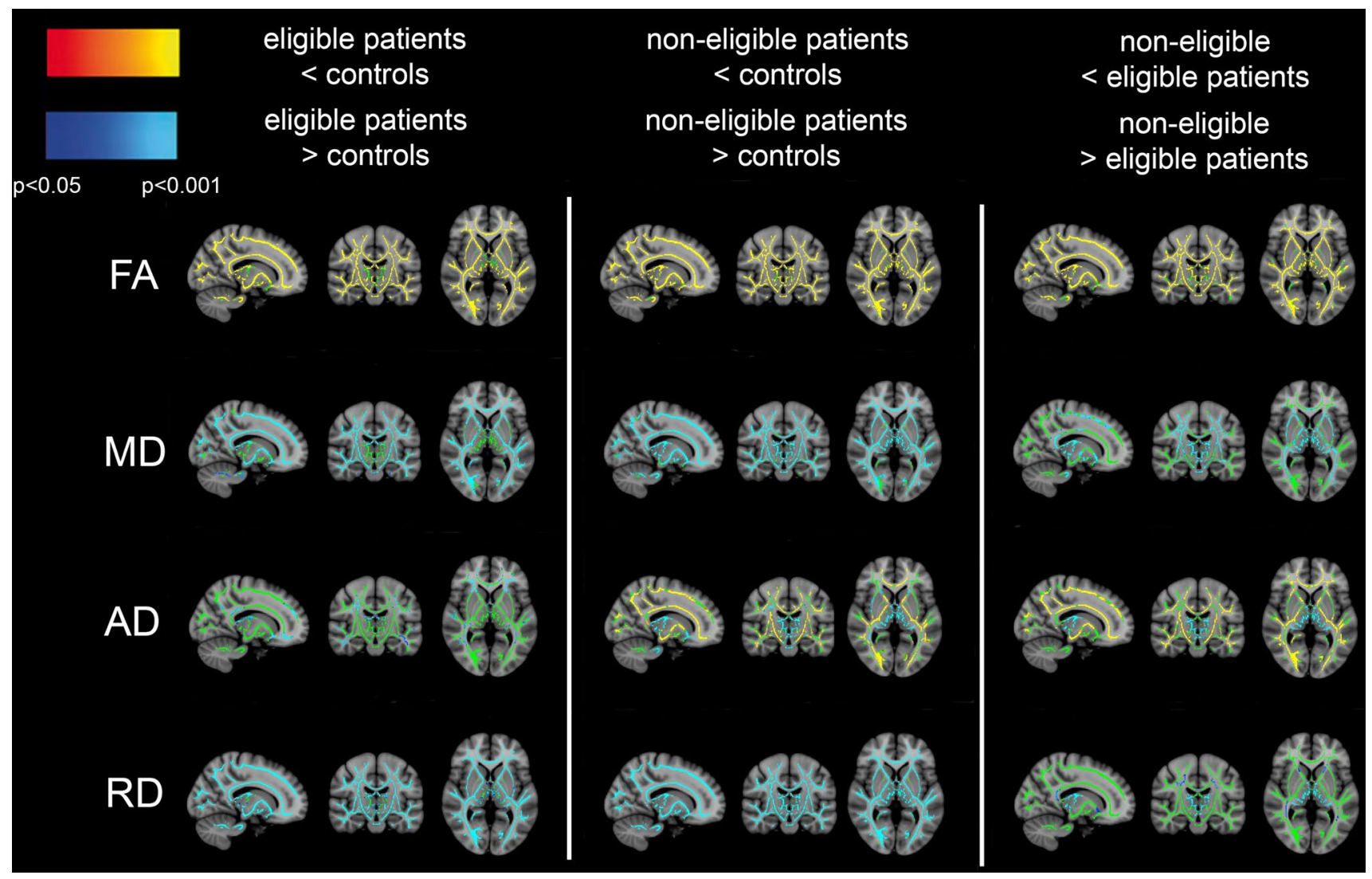

Fig. 1 TBSS analysis for FA, MD, AD and RD comparing HCT-eligible patients vs. control subjects (left column), non-eligible patients vs. control subjects (middle column) and non-eligible vs. eligible patients (right column). FA was decreased (orange-yellow) in eligible and non-eligible patients compared to controls and in non-eligible patients compared to eligible patients in almost the whole skeleton. MD and RD were increased (blue-light blue) in both patient groups compared to controls, and in non-eligible patients compared to eligible patients. $\mathrm{AD}$ was increased in eligible patients compared to con-

Following the TBSS findings, MD and RD were increased in both patient groups in NAWM, corpus callosum, pyramidal tracts and thalamus, and differences were most pronounced between controls and non-eligible patients. Differences in RD were larger than differences in MD, again with the corpus callosum showing most prominent differences between groups. MD and RD within abnormal WM were higher than in NAWM, but did not differ between patient groups (supplementary material, Table 1).

In the pyramidal tracts, there were no group differences in AD. Following the TBSS findings, in NAWM and corpus callosum, AD was lower in both patient groups than in controls, whereas in the thalamus $\mathrm{AD}$ was higher in patients. Again, these differences were most pronounced between HCT-non-eligible patients and controls. AD within abnormal WM was higher than in NAWM, and lower in noneligible patients than in eligible patients (supplementary material, Table 1). trols in parts of the skeleton. When comparing non-eligible patients to controls or to eligible patients, $\mathrm{AD}$ was increased mainly in the thalamus (blue-light blue), and decreased in WM areas including the corpus callosum (orange-yellow). The part of the WM skeleton that does not differ between groups is indicated in green. A family-wise error corrected $p<0.05$ was considered significant. As shown in the color bar, orange and blue correspond to $p<0.05$, while yellow and light blue correspond to $p<0.001$

We observed similar patterns for MD, RD and AD when splitting the groups based on field strength (supplementary material, Fig. 1).

Spearman rank correlations with MLD-GMF were significant for FA ( -0.84$), \mathrm{MD}(0.78)$ and $\mathrm{RD}(0.86)$, all $p<0.01$. Thus, low FA and high MD and RD of the pyramidal tracts at baseline indicate poor motor function at follow-up.

\section{Longitudinal evolvement of diffusion measures}

For illustration, a selection of longitudinal diffusion measures in selected ROIs is shown in Fig. 3. For each patient with follow-up measurements, symbols are connected by lines. The longitudinal variation indicates the actual course, but also the reproducibility of the measurement, including the effect of examinations at both field strengths for some patients. The effect of field strength can also be appreciated when comparing control subjects at 3 and $1.5 \mathrm{~T}$. Overall, 

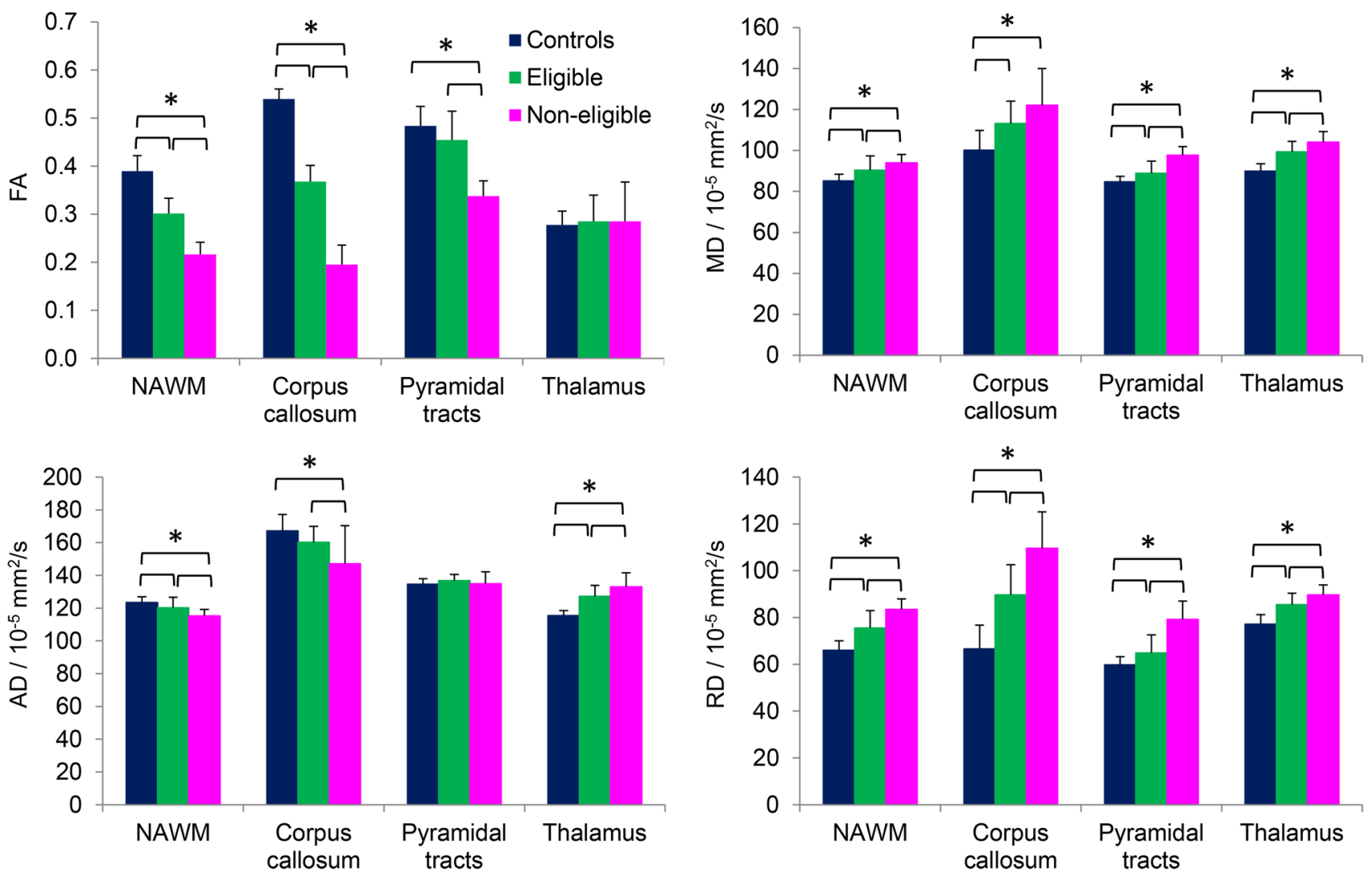

Fig. 2 Mean values for FA, MD, AD and RD in NAWM, corpus callosum, pyramidal tracts and thalamus for control subjects (blue), eligible (green) and non-eligible (pink) patients. Error bars indicate

measures remained relatively stable, especially for HCTeligible patients after treatment. In non-eligible patients, values showed a progressively abnormal trend.

In NAWM, FA mildly fluctuated for treated eligible patients, while FA further decreased for HCT-non-eligible patients (Fig. 3a). Diffusivities remained relatively stable for all patients. In the corpus callosum, FA tended to decrease in the treated eligible patients, while the reduction in the non-eligible patients was marginal (Fig. 3b). However, MD, AD and RD longitudinally increased especially in non-eligible patients, which meant that $\mathrm{AD}$, which was decreased at baseline, showed a pseudo-normalization (Fig. 3c).

In the pyramidal tracts, FA remained constant or slightly increased over time in most treated eligible patients, whereas FA slightly decreased in HCT-non-eligible patients (Fig. 3d). Diffusivities showed some longitudinal variability, but no clear trend was observed.

In the thalamus, in which FA did not differ between groups at baseline, FA remained stable in treated eligible patients, and showed a slight decrease in non-eligible patients (Fig. 3e). Diffusivities in treated eligible patients remained stable or showed a mild increase, whereas a

standard deviations. Significant differences between groups are indicated with square brackets and a single asterisk (post hoc Dunnett's $\mathrm{T} 3, p<0.05)$

larger increase was observed in non-eligible patients (as shown for AD in Fig. 3f).

\section{Discussion}

Using diffusion-weighted MRI, we compared magnitude and direction of diffusion between MLD patients and controls to gain insight into the microstructure of affected brain tissue. At baseline, FA was decreased and MD and RD were increased in MLD patients compared to controls throughout the WM, not only in the corpus callosum (affected early in the disease), but also in NAWM. FA measures of the thalamus did not differ between groups, but its components $\mathrm{AD}$ and $\mathrm{RD}$ were both increased in patients compared to controls. Whereas AD was increased in thalamus, it was unchanged in the pyramidal tracts and decreased in the corpus callosum and, to a lesser degree, in NAWM. All differences were most pronounced between controls and HCTnon-eligible patients.

Longitudinally, in treated HCT-eligible patients, diffusion measures remained stable or showed only minor changes. FA remained constant or even tended to increase in NAWM, 

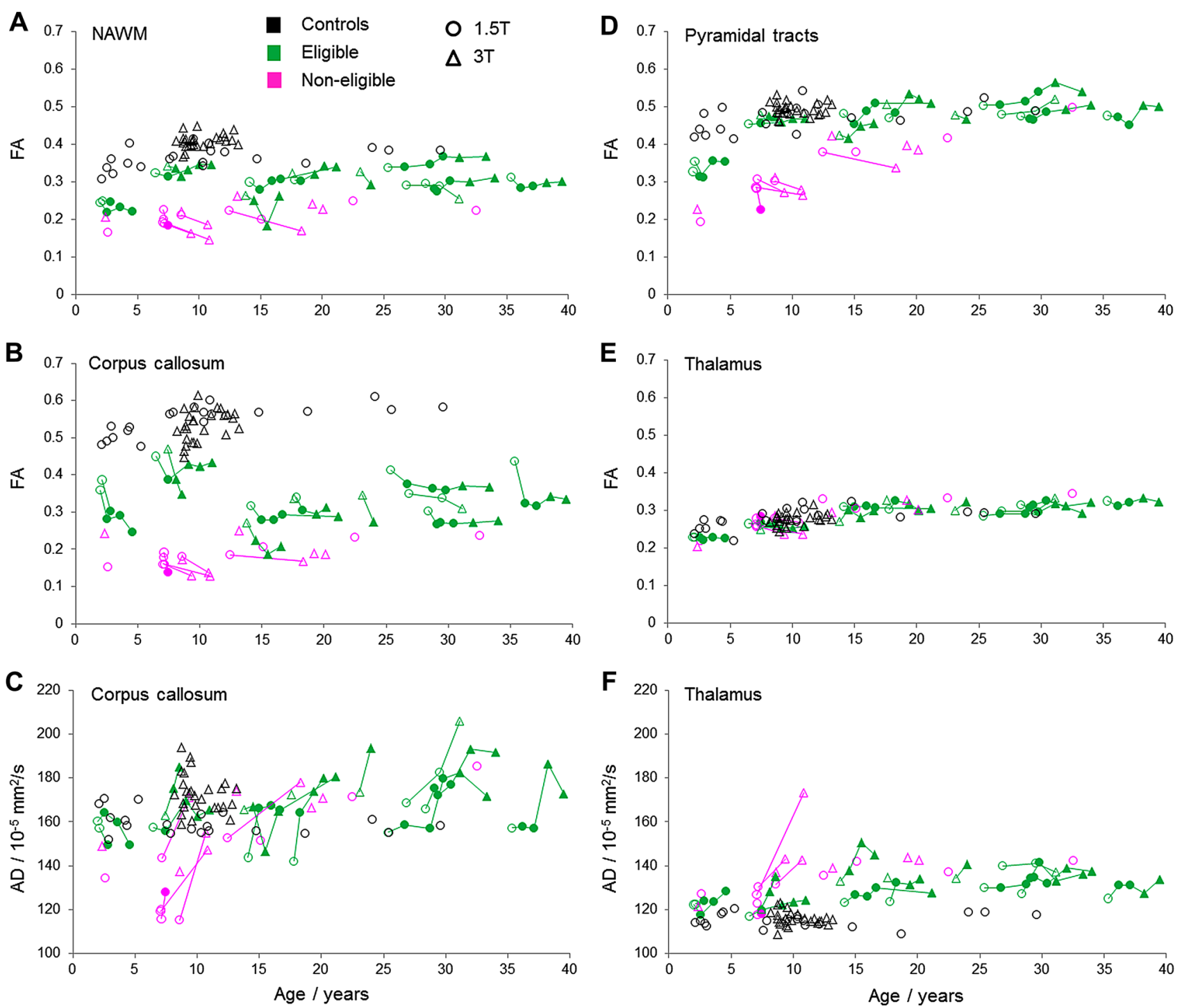

Fig. 3 Longitudinal evolvement of a FA in NAWM, b FA and $\mathbf{c}$ AD in corpus callosum, $\mathbf{d}$ FA in pyramidal tracts, e FA and $\mathbf{f}$ AD in thalamus. Control values are indicated in black, HCT-eligible patients in

pyramidal tracts, and thalamus, whereas it slightly decreased in the corpus callosum. HCT-non-eligible patients had less follow-up examinations than eligible patients, but those available showed clear increases of $\mathrm{RD}$ and $\mathrm{AD}$, causing a small FA reduction in all investigated regions. The treatment effect of HCT most likely influenced the longitudinal differences between treated eligible patients (approaching control values in NAWM and pyramidal tracts), and untreated non-eligible patients (increasingly abnormal values). This is in line with our observation that metabolite concentrations observed with magnetic resonance spectroscopy partially normalized in successfully transplanted patients, whilst concentrations for non-treated patients further deteriorated [30].

green and non-eligible patients in pink. Data measured at $1.5 \mathrm{~T}$ are indicated with a circle, data measured at $3 \mathrm{~T}$ with a triangle. After transplantation, symbols are filled

The diffusion tensor model used in this study reflects the underlying structural characteristics in a simplified manner, hampered by partial volume effects and crossing fibers [18]. Advanced multi-compartment diffusion models, such as the composite hindered and restricted model of diffusion (CHARMED), are more sensitive than the conventional ones [31-33]. However, since our study, ongoing since 2007, concerns a rare disease, application of these advanced diffusion models was not feasible.

This implies that we can merely hypothesize about the precise mechanism responsible for the observed differences rather than draw general conclusions because different cellular processes may lead to identical changes [9-11]. Since both animal $[13,34,35]$ and human studies [36] have 
shown an increased RD parallel to myelin loss, our results of increased RD in WM suggest myelin loss in patients, in line with histopathological findings [37]. This is also supported by our observation that high RD and low FA in the pyramidal tracts at baseline indicate poor motor function at follow-up.

With regard to $\mathrm{AD}$, animal and human studies provide discrepant results, correlating axonal damage with either an AD decrease [13, 17] or increase, [38, 39], respectively. This reflects the difficulty in relating $\mathrm{AD}$ to underlying pathological processes. Our observation of opposing AD patterns in MLD suggests that different pathological mechanisms can cause either a decrease or an increase in $\mathrm{AD}$, with the overall balance between these effects depending on brain region and disease stage. MLD is characterized by accumulation of sulfatides, major myelin lipids mainly synthesized by oligodendrocytes. Analytical studies of MLD patients' brain tissue showed that metachromatic deposits are mainly present in the WM, with a sulfatide content up to eight times higher than normal, with relatively minor chemical GM changes [37]. Regarding WM, we assume that the massive intracellular sulfatide accumulation in swollen macrophages, in vain trying to digest these lipids, causes overall diffusion restriction and thereby AD reduction. Using conventional DWI, restricted diffusion in the outermost part of the demyelinated WM has indeed been described for single cases in relatively early disease stage $[40,41]$. However, as the disease progresses, axons are increasingly damaged. Based on the previous observations in human studies, we expect that loss of both myelin and axons will lead to an AD increase [38, 39]. Our results suggest that, particularly in the corpus callosum of non-eligible patients at baseline, diffusion restriction due to sulfatide accumulation in macrophages contributes more to the severely reduced AD values than increased diffusion due to myelin and axonal loss. Our longitudinal observation of an increase, and thereby a pseudo-normalization of AD, in the corpus callosum suggests that myelin and axonal loss likely becomes more prominent in progressive disease. The corpus callosum is, of the investigated ROIs, least hindered by limitations of the tensor model, suggesting that the interpretation of $\mathrm{AD}$ values is not much influenced by the presence of crossing fibers. Whether inflammation and gliosis also contribute to $\mathrm{AD}$ changes in this setting is uncertain.

The thalamus is a DGM structure, in which sulfatide accumulation is much more limited than in WM [37]. In addition, the thalamus has a different microstructure than $\mathrm{WM}$ as it largely consists of neurons and contains few axons. In control subjects, this is mirrored by low thalamic FA, as the difference between AD and RD is much smaller than for a WM structure like the corpus callosum. In the thalamus of patients, we observed an increase of $\mathrm{AD}$ and RD of similar relative magnitude, which had hardly any effect on FA. This increase of both $\mathrm{AD}$ and RD probably implies an increase in extracellular space due to neuronal loss, which apparently dominates reductions in diffusivity due to storage material.

Limitations of this study were its retrospective character, a large age range of patients (inherent to the inclusion of patients with all disease types), and a limited age range of controls at $3 \mathrm{~T}$. The combination of 1.5 and $3 \mathrm{~T}$ data also introduced some variability, although these differences were typically smaller than differences between controls and patients. In fact, the ROI-based analysis of baseline data at 1.5 and at $3 \mathrm{~T}$ separately (as shown in the supplementary material) showed identical patterns as those observed in the combined analysis. We noticed the same main group effects, although in these smaller groups fewer post hoc pairwise comparisons were significant.

Overall, the observed changes of FA, RD, and especially AD indicate that MLD alters certain aspects of brain microstructure. These changes most likely reflect a multitude of pathological processes such as accumulation of metachromatic material, followed by myelin and axonal loss. The differences between untreated and treated patients indicate that diffusion measures are positively affected by HCT, further emphasizing the beneficial effects of this intervention on WM and supporting the findings of other quantitative MR measures as proton MR spectroscopy [30]. Altogether, quantitative MR measures provide more insight into timedependent disease mechanisms and might in the future aid in determining the right window for intervention.

Acknowledgements The authors thank all the patients with MLD and their families for participating in this study.

Author contributions PJWP and NIW had full access to all the data in the study and take responsibility for the integrity of the data and the accuracy of the data analysis. All authors substantially contributed to conception or design of the work or the acquisition, analysis or interpretation of data. DFR, PJWP and NIW wrote the manuscript. All authors critically revised the manuscript for intellectual content. All authors did a final review and approved the manuscript. All authors agreed to be accountable for all aspects of the work in ensuring that questions related to the accuracy or integrity of any part of the work are appropriately investigated and resolved.

Funding This study was financed by Metakids, a charity sponsoring research on metabolic disorders (Project 2015-059).

\section{Compliance with ethical standards}

Conflicts of interest On behalf of all authors, the corresponding author states that there is no conflict of interest.

Ethical standard The study has been approved by the ethics committee of VU University Medical Center.

Informed consent Informed consent was obtained from the participants or their guardians. 
Open Access This article is distributed under the terms of the Creative Commons Attribution 4.0 International License (http://creativeco mmons.org/licenses/by/4.0/), which permits unrestricted use, distribution, and reproduction in any medium, provided you give appropriate credit to the original author(s) and the source, provide a link to the Creative Commons license, and indicate if changes were made.

\section{References}

1. Eckhardt M (2008) The role and metabolism of sulfatide in the nervous system. Mol Neurobiol 37:93-103

2. van Rappard DF, Boelens JJ, Wolf NI (2015) Metachromatic leukodystrophy: disease spectrum and approaches for treatment. Best Pract Res Clin Endocrinol Metab 29:261-273

3. Groeschel S, Kuhl JS, Bley AE, Kehrer C et al (2016) Longterm outcome of allogeneic hematopoietic stem cell transplantation in patients with juvenile metachromatic leukodystrophy compared with nontransplanted control patients. JAMA Neurol 73:1133-1140

4. van Rappard DF, Boelens JJ, van Egmond ME, Kuball J et al (2016) Efficacy of hematopoietic cell transplantation in metachromatic leukodystrophy: the Dutch experience. Blood 127:3098-3101

5. van der Knaap MS, Valk J (2015) Magnetic resonance of myelination and myelin disorders, 3rd edn. Springer, Berlin

6. Tillema JM, Derks MG, Pouwels PJ, de Graaf Pim et al (2015) Volumetric MRI data correlate to disease severity in metachromatic leukodystrophy. Ann Clin Transl Neurol 2:932-940

7. Martin A, Sevin C, Lazarus C, Bellesme C et al (2012) Toward a better understanding of brain lesions during metachromatic leukodystrophy evolution. Am J Neuroradiol 33:1731-1739

8. van der Voorn JP, Pouwels PJ, Kamphorst W, Powers JM et al (2005) Histopathologic correlates of radial stripes on MR images in lysosomal storage disorders. Am J Neuroradiol 26:442-446

9. Assaf Y, Pasternak O (2008) Diffusion tensor imaging (DTI)based white matter mapping in brain research: a review. J Mol Neurosci 34:51-61

10. Basser PJ, Jones DK (2002) Diffusion-tensor MRI: theory, experimental design and data analysis - a technical review. NMR Biomed 15:456-467

11. Jones DK, Knosche TR, Turner R (2013) White matter integrity, fiber count, and other fallacies: the do's and don'ts of diffusion MRI. Neuroimage 73:239-254

12. Song SK, Sun SW, Ramsbottom MJ, Chang C et al (2002) Dysmyelination revealed through MRI as increased radial (but unchanged axial) diffusion of water. Neuroimage 17:1429-1436

13. Song SK, Yoshino J, Le TQ, Lin SJ et al (2005) Demyelination increases radial diffusivity in corpus callosum of mouse brain. Neuroimage 26:132-140

14. Klawiter EC, Xu J, Naismith RT, Benzinger TL et al (2012) Increased radial diffusivity in spinal cord lesions in neuromyelitis optica compared with multiple sclerosis. Mult Scler 18:1259-1268

15. Alexander AL, Lee JE, Lazar M, Field AS (2007) Diffusion tensor imaging of the brain. Neurotherapeutics 4:316-329

16. Boretius S, Escher A, Dallenga T, Wrzos C et al (2012) Assessment of lesion pathology in a new animal model of MS by multiparametric MRI and DTI. Neuroimage 59:2678-2688

17. Brennan FH, Cowin GJ, Kurniawan ND, Ruitenberg MJ (2013) Longitudinal assessment of white matter pathology in the injured mouse spinal cord through ultra-high field (16.4 T) in vivo diffusion tensor imaging. Neuroimage 82:574-585
18. Wheeler-Kingshott CA, Cercignani M (2009) About, "axial" and "radial" diffusivities. Magn Reson Med 61:1255-1260

19. Kehrer C, Blumenstock G, Raabe C, Krageloh-Mann I (2011) Development and reliability of a classification system for gross motor function in children with metachromatic leucodystrophy. Dev Med Child Neurol 53:156-160

20. Königs M, Pouwels PJ, Ernest van Heurn LW, Bakx R et al (2017) Relevance of neuroimaging for neurocognitive and behavioral outcome after pediatric traumatic brain injury. Brain Imaging Behav. https://doi.org/10.1007/s11682-017-9673-3

21. Steenweg ME, Wolf NI, van Wieringen WN, Barkhof F et al (2016) Quantitative MRI in hypomyelinating disorders: correlation with motor handicap. Neurology 87:752-758

22. Smith SM, Jenkinson M, Johansen-Berg H, Rueckert D et al (2006) Tract-based spatial statistics: voxelwise analysis of multisubject diffusion data. Neuroimage 31:1487-1505

23. Clas P, Groeschel S, Wilke M (2012) A semi-automatic algorithm for determining the demyelination load in metachromatic leukodystrophy. Acad Radiol 19:26-34

24. Chard DT, Jackson JS, Miller DH, Wheeler-Kingshott CA (2010) Reducing the impact of white matter lesions on automated measures of brain gray and white matter volumes. J Magn Reson Imaging 32(1):223-228

25. Zhang Y, Brady M, Smith S (2001) Segmentation of brain MR images through a hidden Markov random field model and the expectation-maximization algorithm. IEEE Trans Med Imaging 20:45-57

26. Patenaude B, Smith SM, Kennedy D, Jenkinson M (2011) A Bayesian model of shape and appearance for subcortical brain segmentation. Neuroimage 56:907-922

27. Hua K, Zhang J, Wakana S, Jiang H et al (2008) Tract probability maps in stereotaxic spaces: analyses of white matter anatomy and tract-specific quantification. Neuroimage 39:336-347

28. Tzourio-Mazoyer N, Landeau B, Papathanassiou D, Crivello F et al (2002) Automated anatomical labeling of activations in SPM using a macroscopic anatomical parcellation of the MNI MRI single-subject brain. Neuroimage 15:273-289

29. Behrens TE, Berg HJ, Jbabdi S, Rushworth MF et al (2007) Probabilistic diffusion tractography with multiple fibre orientations: what can we gain? Neuroimage 34:144-155

30. van Rappard DF, Klauser A, Steenweg ME, Boelens JJ et al (2018) Quantitative MR spectroscopic imaging in metachromatic leukodystrophy: value for prognosis and treatment. J Neurol Neurosurg Psychiatry 89:105-111

31. Harms RL, Fritz FJ, Tobisch A, Goebel R et al (2017) Robust and fast nonlinear optimization of diffusion MRI microstructure models. Neuroimage 155:82-96

32. De Santis S, Drakesmith M, Bells S, Assaf Y et al (2014) Why diffusion tensor MRI does well only some of the time: variance and covariance of white matter tissue microstructure attributes in the living human brain. Neuroimage 89:35-44

33. Assaf Y, Basser PJ (2005) Composite hindered and restricted model of diffusion (CHARMED) MR imaging of the human brain. Neuroimage 27:48-58

34. Hofling AA, Kim JH, Fantz CR, Sands MS et al (2009) Diffusion tensor imaging detects axonal injury and demyelination in the spinal cord and cranial nerves of a murine model of globoid cell leukodystrophy. NMR Biomed 22:1100-1106

35. Ruest T, Holmes WM, Barrie JA, Griffiths IR et al (2011) Highresolution diffusion tensor imaging of fixed brain in a mouse model of Pelizaeus-Merzbacher disease: comparison with quantitative measures of white matter pathology. NMR Biomed 24:1369-1379

36. Schmierer K, Wheeler-Kingshott CA, Tozer DJ, Boulby PA et al (2008) Quantitative magnetic resonance of postmortem 
multiple sclerosis brain before and after fixation. Magn Reson Med 59:268-277

37. Morell P (1984) Myelin, 2nd edn. Plenum Press, North Carolina

38. Hulst HE, Steenwijk MD, Versteeg A, Pouwels PJ et al (2013) Cognitive impairment in MS: impact of white matter integrity, gray matter volume, and lesions. Neurology 80:1025-1032

39. Klistorner A, Vootakuru N, Wang C, Yiannikas C et al (2015) Decoding diffusivity in multiple sclerosis: analysis of optic radiation lesional and non-lesional white matter. PLoS One 10:e0122114

40. Oguz KK, Anlar B, Senbil N, Cila A (2004) Diffusion-weighted imaging findings in juvenile metachromatic leukodystrophy. Neuropediatrics 35:279-282

41. Sener RN (2003) Metachromatic leukodystrophy. Diffusion MR imaging and proton MR spectroscopy. Acta Radiol 44:440-443

\section{Affiliations}

\section{Diane F. van Rappard ${ }^{1,2} \cdot$ Marsh Königs $^{3,4} \cdot$ Marjan E. Steenweg ${ }^{1,2}$. Jaap Jan Boelens ${ }^{5}$. Jaap Oosterlaan ${ }^{3,6}$. Marjo S. van der Knaap ${ }^{1,2,7}$. Nicole I. Wolf ${ }^{1,2}$. Petra J. W. Pouwels ${ }^{2,8}$}

1 Department of Pediatric Neurology, Center for Childhood White Matter Disorders, VU University Medical Center, Amsterdam, The Netherlands

2 Amsterdam Neuroscience, VU University Medical Center Amsterdam, Academic Medical Center, VU University Amsterdam and University of Amsterdam, Amsterdam, The Netherlands

3 Clinical Neuropsychology Section, FGB VU University, Amsterdam, The Netherlands

4 Emma Children's Hospital, Academic Medical Center Amsterdam, Amsterdam, The Netherlands
5 Department of Pediatrics, Blood and Marrow Transplantation Program, University Medical Center Utrecht, Utrecht, The Netherlands

6 Department of Pediatrics, VU University Medical Center Amsterdam, Amsterdam, The Netherlands

7 Department of Functional Genomics, Center for Neurogenomics and Cognitive Research, VU University, Amsterdam, The Netherlands

8 Department of Radiology and Nuclear Medicine, VU University Medical Center, Amsterdam, The Netherlands 